\title{
Denial-of-Service Resistant Bandwidth Allocation for MANETs
}

\author{
Marek Hejmo, Brian L. Mark, Charikleia Zouridaki \\ Department of Electrical and Computer Engineering \\ George Mason University \\ Fairfax, VA 22030 \\ Email: \{mhejmo,bmark,czourida\}@gmu.edu
}

\begin{abstract}
We consider the problem of allocating bandwidth to a set of traffic flows at a statistical multiplexer to provide both quality-of-service $(\mathrm{QoS})$ and resistance to a class of denialof-service (DoS) attacks. The target application is QoS signaling in a mobile ad hoc network (MANET) environment where the channel bandwidth is variable and the mobile device handles bandwidth requests arriving from multi-hop flows. An admission controller maintains a reserved rate to limit the aggregate traffic rate and to make admission decisions. We analyze the behavior of a rate adjustment scheme based on a Markov Modulated Poisson Process (MMPP) model, which captures the flow-level and burst-level characteristics of variable bit rate traffic. We propose a scheme for adjusting the reserved rate using traffic measurements and an MMPP parameter estimation applied to a reduced MMPP model. Finally, we develop a scheme to estimate parameters for a heuristic rate adjustment scheme that can be executed in real-time. We present numerical results that illustrate the modeling approach and demonstrate the effectiveness of the proposed bandwidth allocation schemes.
\end{abstract}

\section{INTRODUCTION}

We consider the problem of allocating bandwidth to a set of traffic flows to provide both quality-of-service (QoS) and resistance to a class of denial-of-service (DoS) attacks. The target application is QoS signaling in a mobile ad hoc network (MANET), although the problem and our proposed solution approach are applicable to other statistical multiplexing scenarios. In the MANET setting, the DoS problem can be especially severe due to the limited bandwidth and time-varying characteristics of the wireless channel, and node mobility. We assume that a mobile node handles bandwidth requests arriving from multi-hop traffic flows. The set of traffic flows is policed as an aggregate traffic stream at the allocated rate, but the flows are not policed individually. Perflow policing is generally neither scalable nor feasible for mobile devices.

We study the problem of selecting the reserved rate using a simplified Markov Modulated Poisson Process (MMPP), which represents the burst-scale and flow-scale characteristics of the aggregate traffic stream. We simplify the traffic model further to a reduced 3-state MMPP, which is then used to derive an offline rate adjustment scheme based on estimation of the MMPP parameters. The offline scheme is used to estimate the parameters of an real-time rate adjustment heuristic introduced in [1]. Our numerical results demonstrate with appropriately chosen parameters, the rate adjustment heuristic can effectively track the profile of a variable bit rate traffic stream and hence provide resistance to flooding and overreservation attacks.

The main objectives of the paper are to study the impact of bandwidth allocation on denial-of-service in QoS provisioning for a statistical multiplexer and to propose a measurementbased scheme for dynamically adjusting the reserved rate to mitigate flooding and overreservation attacks. The remainder of the paper is organized as follows. Section II provides further background and motivation for this work and discusses related work in the literature. Section III presents an MMPP-based traffic model for studying the bandwidth allocation problem and discusses a method for estimating the MMPP parameters. In Section IV, a rate adjustment scheme based on the concept of a guard rate derived from the MMPP traffic model is developed and applied to determine the parameters of a realtime rate adjustment heuristic. Numerical results are presented in Section V. Finally, the paper is concluded in Section VI.

\section{BACKGROUND AND RELATED WORK}

\section{A. Denial-of-service Resistant QoS signaling in MANETs}

In a mobile ad hoc network (MANET), nodes carry intransit traffic flows originating from other nodes. The mobile nodes are expected to provide sufficient resources for traffic flows to maintain their desired quality-of-service (QoS). At the same time, they are highly susceptible to denial-of-service (DoS) attacks launched by malicious traffic flows.

In [1], we proposed a QoS signaling mechanism for MANETs, called DRQoS (Denial-of-service Resistant Quality-of-Service) that provides resistance to a class of DoS attacks including flooding, overreservation, and state-table exhaustion. State-table exhaustion is avoided by aggregating flows at a node on the basis of a common input/output hop pair. The flow aggregates are called in-out traffic streams. Flow aggregation involves a tradeoff between fairness and ease of management (see [2]).

Flooding attacks are mitigated by policing the in-out traffic streams to a rate which we refer to as the reserved rate. Overreservation attacks are handled by decreasing the reserved rate when the measured rate of the traffic stream is detected to be lower than the current reserved rate by a factor $p_{d}$, in which case the amount by which the reserved rate is decreased is determined by another parameter $\alpha_{d}$ (see Algorithm 2). 
Thus, DRQoS bandwidth allocation scheme imposes a "use-it or lose-it" policy parameterized by $p_{d}$ and $\alpha_{d}$. The choice of parameters $p_{d}$ and $\alpha_{d}$ involves a tradeoff between aggressiveness between avoiding overreservation attacks and avoiding potential QoS degradation to flows. For constant bit rate (CBR) traffic, the simple heuristic for adjusting the reserved rate can be effective for a range of parameter values. However, if the traffic flows are variable bit rate (VBR) rather than CBR, the choice of the parameters $p_{d}$ and $\alpha_{d}$ becomes more challenging due to the bursty nature of VBR traffic. Determining the parameters $p_{d}$ and $\alpha_{d}$ for VBR traffic is the main motivation of the present paper.

\section{B. Measurement-based connection admission control}

The present work has connections to prior work on measurement-based admission control (MBAC) algorithms (cf. [3]-[5]), which make admission control decisions based on traffic measurements rather than explicit, a priori specifications of per-flow traffic descriptors. The objective of such schemes is to provide a specified level of QoS to the admitted flows, based on estimates of the traffic flow characteristics (e.g., mean and variance of bit rate).

DoS-resistant bandwidth allocation differs from that of conventional admission control schemes in that the admission controller enforces a policing mechanism on the aggregate stream such that the policing rate is determined in part by the negotiated rates of admitted connections, as well as the measured aggregate traffic rate. Once the guard rate is lowered, existing flows are limited to the lower bandwidth allocation until a new flow is accepted. The goal here is to provide sufficient bandwidth at the negotiated rate levels, but only to the extent that the rate levels are actually being honored, as verified by traffic measurements. Traffic policing applied to an aggregate traffic stream provides protection from flooding attacks launched by flows within the traffic stream as well as overreservation attacks, which may prevent new flows from being admitted even when resources are available.

\section{Performance Model}

In this section, we model an aggregate stream of VBR sources as a Markov Modulated Poisson Process (MMPP). The model provides a basis for developing a DoS-resistant bandwidth allocation scheme as discussed in Section IV. We then propose a reduced MMPP model and discuss how to estimate the parameters of the reduced model.

\section{A. MMPP traffic model}

We model the arrival of flows to a statistical multiplexer using a finite source model with $N$ sources. The holding time for each flow is assumed to be exponentially distributed with mean $1 / \mu$. The flow inter-generation times are independent and exponentially distributed with mean $1 / \lambda$. Each flow is modeled as a two-state, on-off MMPP to characterize the bursty nature of VBR traffic. In the off state, the flow is silent, where in the on state the flow generates packets according to a Poisson process with rate $r$. The mean time in the off-state is assumed to be exponentially distributed with mean $1 / \beta$, while the mean time in the on-state is exponentially distributed with mean $1 / \alpha$. The on-off MMPP is also called an Interrupted Poisson Process (IPP). The superposition of $k$ such sources is an MMPP with $k+1$ states.

Incorporating the finite source model for the flow arrival process, we obtain an MMPP model consisting of an underlying Markov chain of $(N+1)(N+2) / 2$ states, as illustrated in the state-transition diagram of Fig. 1. Each state is represented by a pair $(i, j)$, where $i$ is the number of active flows at the multiplexer and $j$ is the number of active flows in the on-state, where $0 \leq i \leq N$ and $0 \leq j \leq i$. Equivalently, the underlying Markov chain of the MMPP can be characterized by an infinitesimal generator $Q=\left[Q_{(i, j) ;\left(i^{\prime}, j^{\prime}\right)}\right]$. With reference to Fig. 1, the transition rates can be specified as follows:

- Vertical: low-to-high and high-to-low

$$
\begin{aligned}
& Q_{(i, j),(i+1, j)}=\frac{\lambda \beta}{\alpha+\beta}, \quad 0 \leq i \leq N-1,0 \leq j \leq i ; \\
& Q_{(i, j),(i-1, j)}=(i-j) \mu, \quad 1 \leq i \leq N, 0 \leq j<i .
\end{aligned}
$$

- Horizontal: left-to-right and right-to-left

$$
\begin{aligned}
& Q_{(i, j),(i, j+1)}=(N-j) \alpha, \quad 1 \leq i \leq N ; 0 \leq j<i \\
& Q_{(i, j),(i, j-1)}=j \beta, \quad 1 \leq i \leq N ; 1 \leq j \leq i .
\end{aligned}
$$

- Diagonal: lower-left to upper-right and upper-right to lower-left

$$
\begin{aligned}
& Q_{(i, j),(i+1, j+1)}=\frac{\lambda \alpha}{\alpha+\beta}, \quad 0 \leq i \leq N-1 ; 0 \leq j<i ; \\
& Q_{(i, j),(i-1, j-1)}=j \mu ; \quad 1 \leq i \leq N ; 1 \leq j \leq i ;
\end{aligned}
$$

All other non-diagonal elements of $Q$ are zero. Since each row of the matrix $Q$ sums to zero, the diagonal elements can be specified as follows:

$$
Q_{(i, j) ;(i, j)}=-\sum_{\left(i^{\prime}, j^{\prime}\right) \neq(i, j)} Q_{(i, j) ;\left(i^{\prime}, j^{\prime}\right)} .
$$

The MMPP traffic model is completely specified by the matrix $Q$ and a diagonal rate matrix $\Lambda=\operatorname{diag}\left(r_{(i, j)}\right)$, where $r_{(i, j)} \triangleq j r$. Given the generator matrix $Q$, the equilibium state probability row vector $\boldsymbol{\pi}=\left[\pi_{(i, j)}\right]$ is determined as the solution to the equations

$$
\boldsymbol{\pi} Q=\mathbf{0}, \quad \boldsymbol{\pi} \mathbf{1}=1,
$$

where $\mathbf{0}$ is a row vector of all zeros and $\mathbf{1}$ is a column vector of all ones. Let $S(t)=(I(t), J(t))$ denote the state process of the modulating Markov chain. Then the Poisson rate of the traffic model at time $t$ is given by $R(t)=r J(t)$. We refer to $\{R(t)\}$ as the rate process.

\section{B. Guard rate and packet loss ratio}

Suppose that the aggregate traffic stream is policed at the guard rate $G$, where $0<G<r N$. We say that a guard rate violation occurs if the rate of the traffic stream exceeds $G$. 


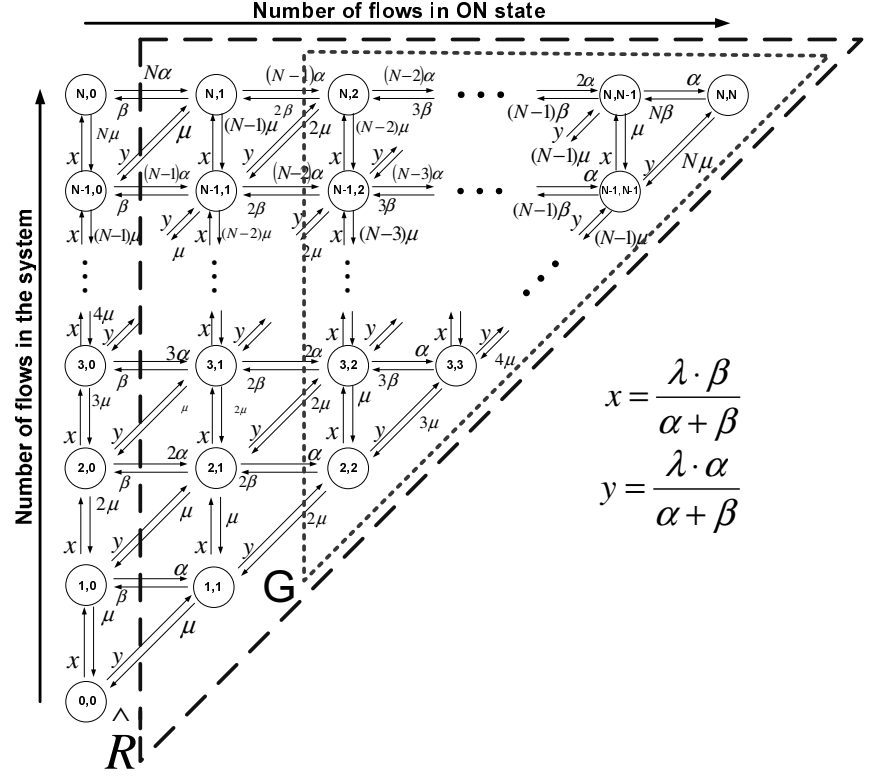

Fig. 1. MMPP traffic model.

More precisely, given the value $G$, the set of states violating the guard bandwidth is given by

$$
\mathcal{S}_{v}(G)=\{(i, j): j r>G\} .
$$

We now introduce a dynamic guard rate, which depends on the measured traffic rate $\hat{R}$ over a sliding observation window. First, we define a conditional packet loss ratio as follows:

$$
L(G \mid \hat{R}) \triangleq \frac{E\left[(R-G)^{+} \mid R \geq \hat{R}\right]}{E[R]},
$$

with

$$
E\left[(R-G)^{+} \mid R \geq \hat{R}\right]=\frac{\sum_{(i, j) \in \triangle(G)}(r j-G) \pi_{(i, j)}}{\sum_{(i, j) \in \triangle(\hat{R})} \pi_{(i, j)}},
$$

where

$$
\triangle(x) \triangleq\{(i, j): r j>x\} .
$$

As can be seen from Fig. $1, \triangle(G)$ and $\triangle(\hat{R})$ are, the sets of states contained in the triangles bounded by $G$ and $\hat{R}$, respectively. The dynamic guard rate is defined in terms of the conditional packet loss ratio as follows:

$$
G(\epsilon \mid \hat{R}) \triangleq \min \{g: L(g \mid \hat{R}) \leq \epsilon\},
$$

where $\epsilon>0$ is a bound on the maximum tolerable packet loss ratio.

\section{Reduced 3-state MMPP model}

The MMPP traffic model described in Section III-A is rather complex as a model for an unknown traffic stream. Moreover, this MMPP carries some fairly specific assumptions about the nature of the VBR flows. For these reasons, we consider a reduced 3-state MMPP traffic model for approximately characterizing an unknown traffic stream. The birth rate in state $i$ is denoted by $\alpha_{i}$ for $i=1,2$, while the death rate in state $i$ is denoted by $\beta_{i}$ for $i=2,3$. The corresponding infinitesimal generator is given by

$$
\tilde{Q}=\left[\begin{array}{ccc}
-\alpha_{1} & \alpha_{1} & 0 \\
\beta_{2} & -\beta_{2}-\alpha_{2} & \alpha_{2} \\
0 & \beta_{3} & -\beta_{3}
\end{array}\right] .
$$

We denote the rate matrix of the MMPP as

$$
\tilde{\Lambda}=\operatorname{diag}\left(\tilde{\lambda}_{1}, \tilde{\lambda}_{2}, \tilde{\lambda}_{3}\right),
$$

where $\tilde{\lambda}_{i}$ is the Poisson arrival rate in state $i, i=1,2,3$. Given the parameters $(\tilde{Q}, \tilde{\Lambda})$ of the 3 -state MMPP, the equilibrium probability state vector $\tilde{\pi}=\left[\tilde{\pi}_{1}, \tilde{\pi}_{2}, \tilde{\pi}_{3}\right]$ can be calculated straightforwardly (cf. (2)).

The MMPP traffic model of Section III-A can be reduced straightforwardly to the 3 -state model by mapping the states of the original model into three sets. Let $M=\lceil(N+1) / 3\rceil$ and $k=(N+1) \bmod 3$. Then the three sets can be defined as follows ${ }^{1}$ :

$$
\begin{aligned}
& \mathcal{S}(1)=\{(i, j): 0 \leq j \leq M-1+I[k=2]\} \\
& \mathcal{S}(2)=\{(i, j): M \leq j \leq 2 M-1+I[k=1]\} \\
& \mathcal{S}(3)=\{(i, j): 2 M \leq j \leq 2 M\}+I[k=2]\},
\end{aligned}
$$

where the set $\mathcal{S}(l)$ corresponds to state $l$ in the 3 -state MMPP and $I[A]$ denotes the indicator function on the condition $A$. The parameters $(Q, \Lambda)$ of the full MMPP model can then be mapped to parameters $(\tilde{Q}, \tilde{\Lambda})$ of the reduced model. The transition rates of the reduced model are given by

$$
\begin{aligned}
& \alpha_{1}=\sum_{s \in \mathcal{S}(1), s^{\prime} \in \mathcal{S}(2)} Q_{s ; s^{\prime}}, \quad \alpha_{2}=\sum_{s \in \mathcal{S}(2), s^{\prime} \in \mathcal{S}(3)} Q_{s ; s^{\prime}} \\
& \beta_{2}=\sum_{s \in \mathcal{S}(2), s^{\prime} \in \mathcal{S}(1)} Q_{s ; s^{\prime}}, \quad \beta_{3}=\sum_{s \in \mathcal{S}(3), s^{\prime} \in \mathcal{S}(2)} Q_{s ; s^{\prime}}
\end{aligned}
$$

The Poisson arrival rates of the reduced model are given by

$$
\tilde{\lambda}_{l}=\frac{\sum_{(i, j) \in \mathcal{S}(l)} r j}{\sum_{s \in \mathcal{S}(l)} \pi_{s}}
$$

for $l=1,2,3$.

The conditional packet loss ratio for the 3-state MMPP model, as a function of the guard rate $G$ and the measured rate $\hat{R}$, can be computed as follows:

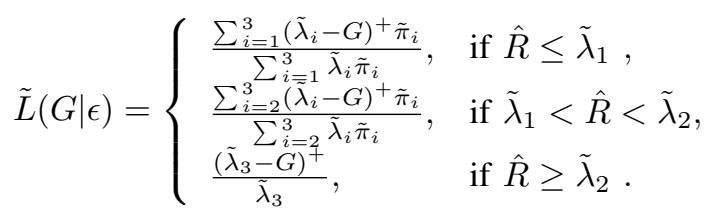

The corresponding dynamic guard rate $\tilde{G}_{d}(\epsilon \mid R)$ is then defined by (cf. (7))

$$
G_{d}(\epsilon \mid \hat{R}) \triangleq \min \{g: L(g \mid \hat{R}) \leq \epsilon\} .
$$

\footnotetext{
${ }^{1}$ The choice of the three sets is not unique.
} 


\section{MMPP parameter estimation}

A given traffic stream can be approximated using the 3-state MMPP model discussed above. The 3-state model consists of seven unknown parameters:

$$
\left(\alpha_{1}, \alpha_{2}, \beta_{2}, \beta_{3}, \tilde{\lambda}_{1}, \tilde{\lambda}_{2}, \tilde{\lambda}_{3}\right) .
$$

Given these parameters, and the measured traffic rate $\hat{R}$, the dynamic guard rate can be computed using (13).

Several methods for MMPP parameter estimation have been proposed in the literature [6], [7]. In our numerical studies, we have adopted a recent approach proposed in [8], which is based on Ryden's method [7] but is more computationally efficient and numerically stable. Ryden's method falls under the class of Expectation-Maximization (EM) algorithms [9].

Ryden's algorithm assumes that the order of the MMPP is known and that an initial starting parameter set $\left(Q^{(0)}, \Lambda^{(0)}\right)$ is given. Given a set of inter-arrival times over an observation window, Ryden's algorithm computes the parameter set $\left(Q^{(1)}, \Lambda^{(1)}\right)$. The parameter set $\left(Q^{(1)}, \Lambda^{(1)}\right)$ is then used as the starting parameter set for the next iteration, which is applied over the same observation window of inter-arrival times. The iterative algorithm is stopped when a termination condition is satisfied.

\section{RATE Adjustment}

Unfortunately, none of the known MMPP estimation techniques, including Ryden's method, is sufficiently efficient to be performed in real-time. In this section, we introduce a rate adjustment scheme based on computing a dynamic guard rate using knowledge of the MMPP parameters. Since the MMPP parameters cannot be estimated in real-time, we apply this algorithm off-line on a set of inter-arrival data to estimate appropriate values for the parameters used in the heuristic rate adjustment algorithm originally proposed in [1].

\section{A. MMPP-based rate adjustment}

Given a traffic stream represented by a sequence of interarrival times, Ryden's method for estimating the MMPP parameters can be applied over a sliding observation window to characterize the traffic stream in terms of the 3-state MMPP model discussed in Section III-C. Using the MMPP parameters $(\tilde{Q}, \tilde{\Lambda})$ for the 3 -state MMPP, the dynamic guard bandwidth $\tilde{G}_{d}$ can be calculated according to (13).

The dynamic guard rate, together with the measured traffic rate $\hat{R}$, can then be used to adjust the reserved rate $R_{r e s}$, as shown in Algorithm 1. Algorithm 1 reduces the reserved rate $R_{\text {res }}$ by a function of the dynamic guard rate $\tilde{G}_{d}$. If the $\tilde{G}_{d}<R_{\text {res }}$, the reserved rate is updated as an average of the current value $R_{\text {res }}$ and the guard rate. The parameter $\gamma \in(0,1)$, used in line 2 of Algorithm 1 , determines the relative weights applied to $R_{r e s}$ and $G_{d}$ in updating the value of $R_{\text {res }}$. In our numerical studies, we have set the value of $\gamma$ to be in the neighborhood of 0.5 . Our numerical results show that Algorithm 1, performs well in terms of meeting the target packet loss rate criterion. However, as discussed earlier, MMPP parameter estimation cannot be executed in real-time.

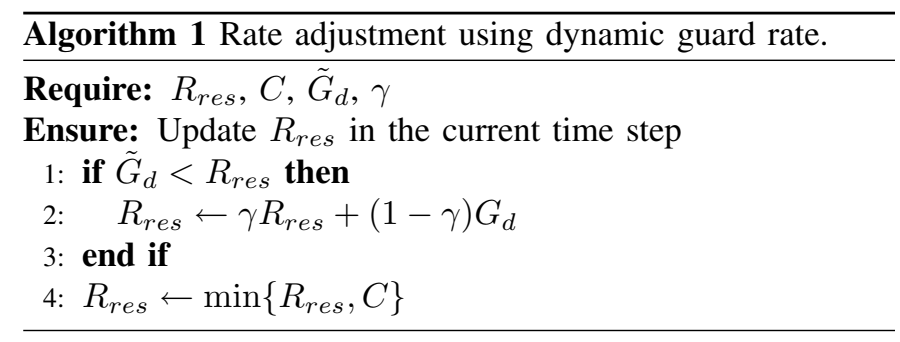

\section{B. DRQoS rate adjustment}

The DRQoS rate adjustment algorithm proposed in [1] and shown in Algorithm 2 can be applied to a real-time traffic stream. However, the algorithm depends strongly on two parameters $p_{d}$ and $\alpha_{d}$ and it is not obvious how these parameters should be set. The reserved rate $R_{\text {res }}$ is updated in a given time interval based on the value of the measured rate $\hat{R}$. If the measured rate $\hat{R}$ is less than $\left(1-p_{d}\right) R_{r e s}$, the reserved rate $R_{\text {res }}$ is reduced by the factor $\left(1-\alpha_{d} p_{d}\right)$.

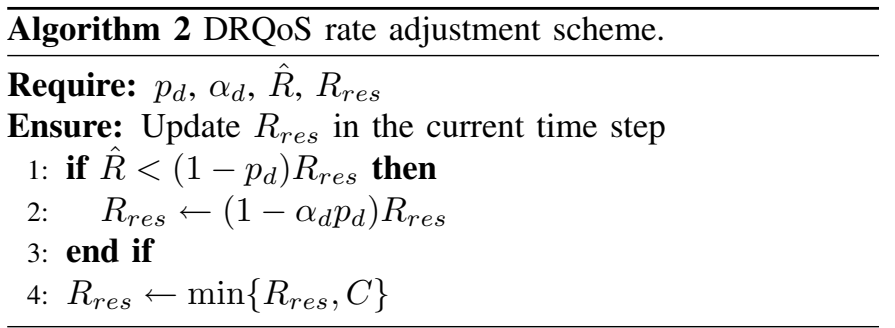

We now propose a method for determining appropriate values for the parameters $\alpha_{d}$ and $p_{d}$ in the DRQoS rate adjustment algorithm based the dynamic guard rate. The method is based on an analogy between Algorithms 1 and 2 . Comparing line 1 of Algorithms 1 and 2, we equate $\tilde{G}_{d}$ and $\hat{R} /\left(1-p_{d}\right)$. Solving for $p_{d}$ yields

$$
p_{d}=1-\hat{R} / \tilde{G}_{d}
$$

Similarly, comparing line 2 in Algorithms 1 and 2, we derive the following equation for $\alpha_{d}$ in terms of $\tilde{G}_{d}, R_{r e s}$, and $p_{d}$ :

$$
\alpha_{d}=\left[1-\left(\gamma+(1-\gamma) \tilde{G}_{d} / R_{r e s}\right)\right] / p_{d}
$$

Averaged values for $p_{d}$ and $\alpha_{d}$ can be computed over a representative traffic stream by inserting (14) and (15) into Algorithm 1. The resulting method for computing $p_{d}$ and $\alpha_{d}$ is shown in Algorithm 3. In our numerical studies we have set the averaging parameter $\eta$ to 0.5 .

\section{NumericAl RESUlts}

In this section, we present simulation results that illustrate the performance of the proposed DoS-resistant bandwidth allocation schemes. In the simulations, the mean flow interarrival time is set as $\lambda^{-1}=1 \mathrm{~s}$ and the mean flow holding time is set as $\mu^{-1}=25 \mathrm{~s}$. Each of the simulation runs is executed over an interval of 3,000 s. In simulation experiments involving the full MMPP model discussed in Section III-A, the total number of sources is set to $N=30$. The peak 

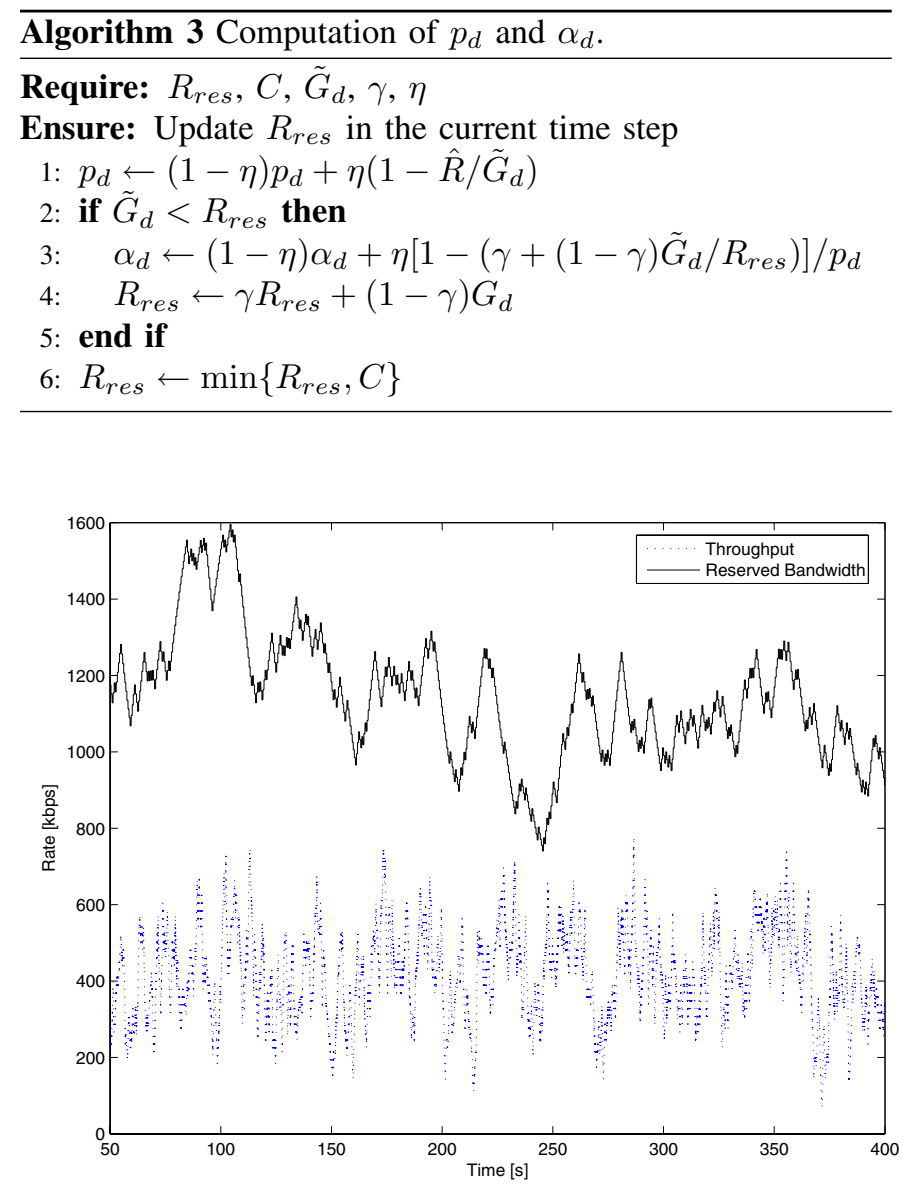

Fig. 2. DRQoS rate adjustment: $p_{d}=0.1, \alpha_{d}=0.2$

rate of each flow is set to $r=50 \mathrm{Kbps}$. For a given flow, the mean on-time is set as $\alpha^{-1}=2 \mathrm{~s}$ and the mean offtime is set as $\beta^{-1}=5 \mathrm{~s}$. A rate adjustment mechanism is triggered once every $T=0.5 \mathrm{~s}$. The rate $\hat{R}$ is estimated using a sliding observation window of length $W=0.4 \mathrm{~s}$ and with step $\Delta=0.04$ s.

Figure 2 shows the aggregate data rate and the reserved rate, when the DRQoS rate adjustment mechanism in Algorithm 2 is used with a conservative parameter setting, i.e., $p_{d}=0.1$ and $\alpha_{d}=0.2$. As one can see, the reserved bandwidth is much higher than the actual data rate of the stream. Thus, the system is vulnerable to over-reservation attacks. The rate adjustment scheme follows the actual data stream more closely when $\alpha_{d}=0.95$, but the packet loss ratio is rather high, at $5.6 \%$ (see Table I).

Figure 3 shows the data rate and the reserved rate when the rate adjustment scheme based on the dynamic guard rate (see Algorithm 1) is applied to the full MMPP model. For this scenarios, we have set the peak rate of each flow to $r=$ $250 \mathrm{Kbps}$. The dynamic guard rate is computed using (7), the target packet loss ratio is set as $\epsilon=0.5 \%$, and the averaging parameter is set as $\gamma=0.5$. As shown in Table II, the actual packet loss ratio that is achieved is $0.017 \%$. Figure 4 shows the data rate and reserved rate when the full MMPP model is

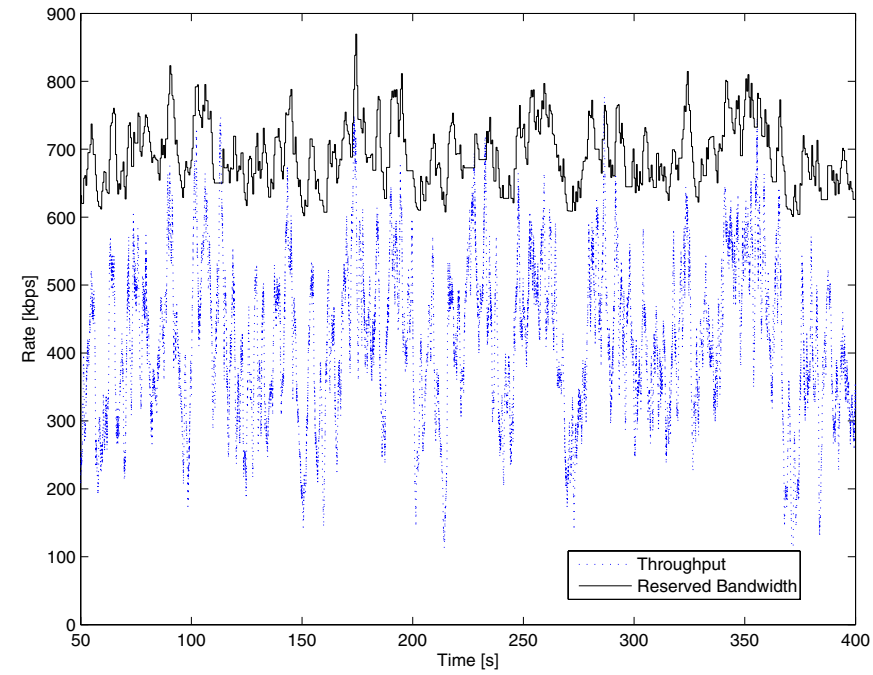

Fig. 3. Rate adjustment for full MMPP model.

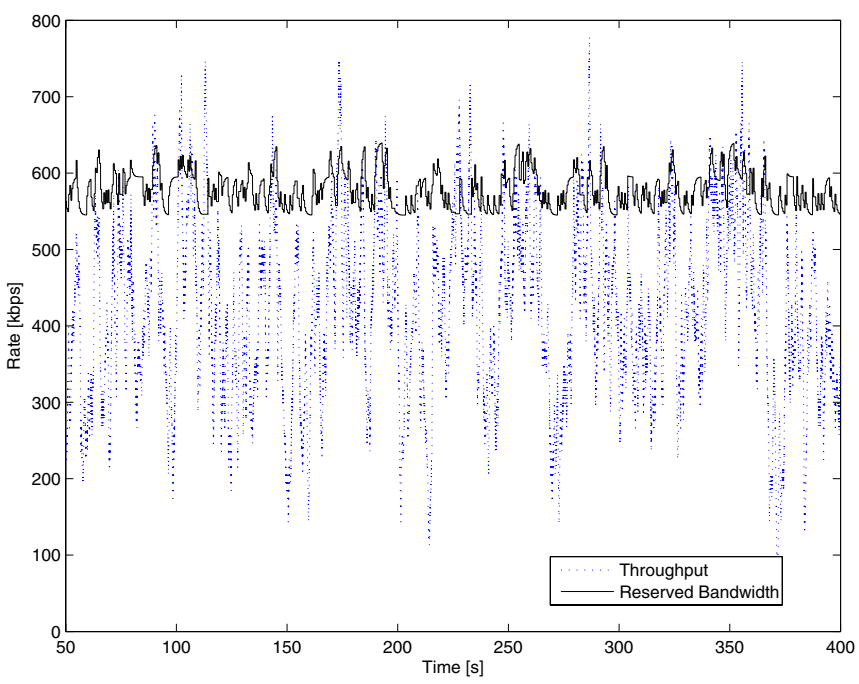

Fig. 4. Rate adjustment for reduced 3-state MMPP.

reduced to the 3-state MMPP using the method described in Section III-C. In this case, the dynamic guard rate is calculated using (13). With the target packet loss ratio of $0.5 \%$, the actual packet loss ratio achieved is $0.425 \%$.

Figure 5 shows data rate and reserved rate curves obtained without knowledge of the parameters of the full MMPP model. Instead the MMPP parameters are estimated using the approach discussed in Section III-D. In this case, the actual packet loss ratio is $3.16 \%$. This suggests that the rate adjustment scheme is not sufficiently conservative to achieve the target loss ratio. Slightly increasing the value of the averaging parameter $\gamma$ from 0.5 to 0.6 , turns out to be sufficient to achieve the target loss ratio.

Finally, Fig. 6 shows the data rate and reserved rate curves obtained using the DRQoS rate adjustment scheme with the 




Fig. 5. Rate adjustment with 3-state MMPP estimation.



Fig. 6. DRQoS rate adjustment with parameters from Algorithm 3.

parameters set as $\alpha_{d}=0.3364$ and $p_{d}=0.2778$. These parameter values were obtained using the method in Algorithm 3, with the averaging parameter $\eta$ set to 0.5 . In this case, the actual packet loss ratio was $0.4074 \%$. Table I summarizes the packet loss ratios obtained using the DRQoS rate adjustment method for various parameter settings. Table II summarizes the packet loss ratios obtained using the guard-based rate adjustment method for the three traffic models corresponding to Figs. 3, 4, and 5, respectively.

\section{CONCLUSION}

We study the problem of denial-of-service (DoS) resistant bandwidth allocation for MANETs using an MMPP-based model of an aggregate traffic stream. We propose an offline algorithm for determining the reserved rate allocation for the

\begin{tabular}{|l|l||l|}
\hline $\boldsymbol{p}_{\boldsymbol{d}}$ & $\boldsymbol{\alpha}$ & Loss ratio [\%] \\
\hline \hline 0.1 & 0.2 & 0.0095 \\
0.1 & 0.95 & 5.61 \\
0.2778 & 0.3364 & 0.4074 \\
\hline
\end{tabular}

TABLE I

DRQOS RATE ADJUSTMENT PERFORMANCE FOR DIFFERENT PARAMETER SETTINGS.

\begin{tabular}{|l|l||l|}
\hline Traffic Model & $\boldsymbol{\epsilon}[\%]$ & Loss ratio [\%] \\
\hline \hline Full MMPP & 0.5 & 0.017 \\
Reduced 3-state MMPP & 0.5 & 0.425 \\
Estimated 3-state MMPP & 0.5 & 3.1642 \\
\hline
\end{tabular}

TABLE II

RATE ADJUSTMENT PERFORMANCE FOR DIFFERENT TRAFFIC MODELS.

traffic stream based on traffic measurements and a method for estimating the parameters of a 3-state MMPP model. We use this algorithm to estimate the parameters for the real-time rate adjustment mechanism proposed in [1]. Our numerical results illustrate the effectiveness of the proposed DoS-resistant bandwidth allocation scheme for an MMPPbased traffic model of an aggregate stream of VBR flows.

\section{ACKNOWLEDGMENT}

The authors thank Prof. Y. Ephraim for helpful discussions on MMPP estimation algorithms. This work was supported in part by the U.S. National Science Foundation under Grant No. CCR-0209049 and Grant No. CCF-0133390.

\section{REFERENCES}

[1] M. Hejmo, B. L. Mark, C. Zouridaki, and R. K. Thomas, "Design and analysis of a denial-of-service resistant quality-of-service signaling protocol for MANETs," IEEE Trans. on Vehicular Technology, vol. 55, no. 3, pp. 743-751, May 2006.

[2] — "On the fairness of flow aggregation for denial-of-service resistant QoS in MANETs," in Proc. IEEE/ACM Workshop on Quality-of-Service in Heterogeneous Wireless/Wired Networks (QShine), Waterloo, Canada, Aug. 2006.

[3] L. Breslau, S. Jamin, and S. Shenker, "Comments on the performance of measurement-based admission control algorithms," in Proc. IEEE Infocom, Tel Aviv, Israel, March 2000.

[4] M. Grossglauser and D. N. C. Tse, "A framework for robust measurementbased admission control," IEEE/ACM Trans. on Networking, vol. 7, no. 3, June 1999.

[5] S. Jamin, P. B. Danzig, S. Shenker, and L. Zhang, "A measurement-based admission control algorithm for integrated services packet networks," IEEE/ACM Transactions on Networking, vol. 5, Feb, 1997.

[6] L. Deng and J. W. Mark, "Parameter estimation for Markov modulated Poisson processes via the EM algorithm with time discretization," Telecommunication Systems, vol. 1, no. 3, pp. 321-338, 1993.

[7] T. Ryden, "An EM algorithm for estimation in Markov-modulated Poisson processes," ELSEVIER Computational Statistics and Data Analysis, vol. 21, pp. 431-447, 1996.

[8] W. J. J. Roberts, Y. Ephraim, and E. Dieguez, “On Ryden's EM algorithm for estimating MMPPs,” IEEE Sig. Proc. Let., vol. 13, pp. 373-376, June 2006.

[9] L. E. Baum, T. Peterie, G. Souled, and N. Weiss, "A maximization technique occurring in the statistical analysis of probabilistic functions of Markov chains," Ann. Math. Statist., vol. 41, no. 1, pp. 164-171, 1970. 